\title{
Examination of Anterior Leaflet Pseudoprolapse Causing Severe Mitral Regurgitation and Its Ideal Surgical Procedure
}

\author{
Chiharu Tanaka, MD, PhD, ${ }^{1}$ Kazumi Akasaka, MD, PhD, ${ }^{2}$ Ryohei Ushioda, $\mathrm{MD},{ }^{1}$ Tomoki Nakatsu, $\mathrm{MD},{ }^{1}$ \\ Naohiro Wakabayashi, MD,${ }^{1}$ Hayato Ise, MD,${ }^{1}$ Hiroto Kitahara, MD,${ }^{1}$ Sentaro Nakanishi, MD, ${ }^{1}$ \\ Natsuya Ishikawa, MD, ${ }^{1}$ Hiroyuki Kamiya, $\mathrm{MD}, \mathrm{PhD}^{1}$ \\ ${ }^{1}$ Department of Cardiac Surgery, Asahikawa Medical University, Asahikawa, Japan; ${ }^{2}$ Medical Laboratory and Blood Center, Asahikawa \\ Medical University Hospital, Asahikawa, Japan
}

\section{ABSTRACT}

Background: The aim of this study is to evaluate severe mitral regurgitation caused by so called atrial leaflet "pseudoprolapse" and verify the effect of simple annular stabilization.

Methods: One-hundred-twenty-two patients underwent surgery for severe mitral regurgitation at our institute between January 2015 to July 2018. Of those, 32 cases diagnosed as anterior leaflet prolapse that underwent mitral repair were analyzed. Ten cases with pseudoprolapse, which is defined as anterior leaflet prolapse without dropping into the left atrium beyond the annular line causing eccentric regurgitation flow directed to the posterior atrium, were classified as the Pseudoprolapse Group. The other 22 cases had obvious anterior leaflet prolapse dropping into the left atrium; these cases were classified as the True Prolapse Group. We compared clinical findings between the 2 groups and reviewed pseudoprolapse cases.

Results: Patients in the Pseudoprolapse Group had lower ejection fraction and lower regurgitation volume than those in the True Prolapse Group. A2 lesion as main inflow of regurgitation was more included in the Pseudoprolapse Group. All but one patient in the Pseudoprolapse Group received only simple annuloplasty, and all patients in the True Prolapse Group received leaflet repair and annuloplasty. In both groups, mid-term regurgitation grade and the reoperation rate were satisfactory. In the Pseudoprolapse Group, 6 cases were clarified as atrial functional mitral regurgitation, and 4 cases were considered to have focal posterior leaflet tethering.

Conclusions: Pseudoprolapse cases could be characterized by low ejection fraction, low regurgitation volume, and A2 prolapse. For most cases with pseudoprolapse, simple annuloplasty may be enough, however further study is needed.

Received December 29, 2019; received in revised form March 4, 2020; accepted March 4, 2020.

Correspondence: Hiroyuki Kamiya, MD, PbD, Department of Cardiac Surgery, Asabikawa Medical University, Midorigaoka Higashi 2-1-1-1, Asabikawa 078-8510, Japan; +81-166-68-2490, fax: +81-166-68-2499 (e-mail: kamiya@asabikawa-med.ac.jp).

\section{INTRODUCTION}

Anterior mitral leaflet (AML) prolapse causes eccentric mitral regurgitation (MR) flow directed to the posterior atrium, due to the gap created by the leaflets. However, the AML prolapse without anterior chordal rupture or elongation does not always need mitral valve reconstruction. We speculated these cases could be considered as "pseudoprolapse," which is defined by the gap created by the leaflets without dropping into the left atrium (LA) beyond the anteroposterior annular line with eccentric MR flow directed to the posterior atrium. Berdeio et al and Hashim et al coined the term "pseudoprolapse" for such cases regarding the ischemic functional MR [Hashim 2012; Berdejo 2017]. Pseudoprolapse might include atrial functional MR (AFMR),

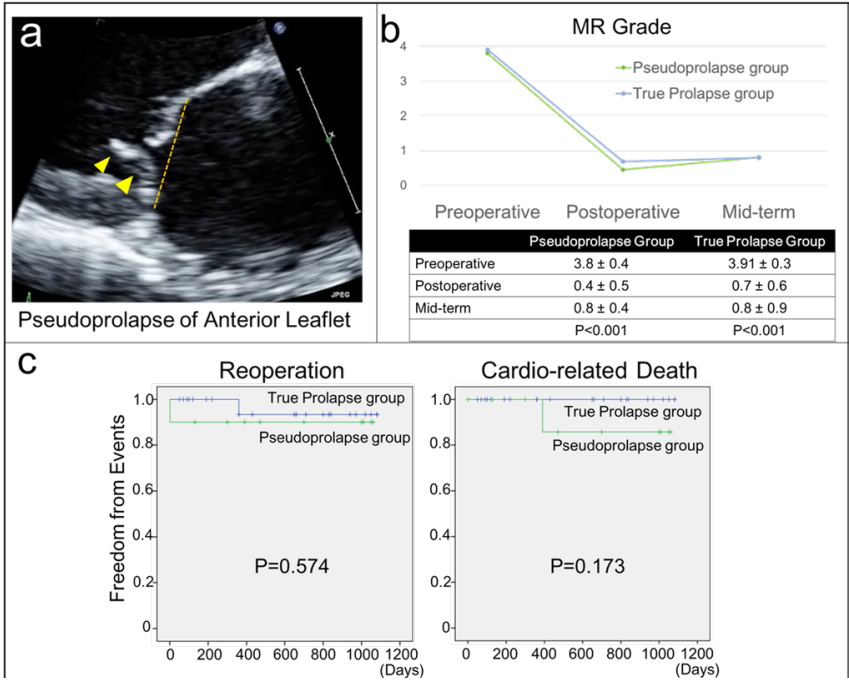

Figure 1. A) Echocardiographic finding of pseudoprolapse of AML. The mitral leaflets create gap each other without dropping into the LA beyond the anteroposterior annular line, which is indicated by the yellow line. The PML causes tethering (as indicated by yellow delta) and results in the AML seeming to be prolapsed; B) MR grade preoperatively, postoperatively, and in the mid-term period. MR grades of the PP Group and the TP Group were improved after surgery, and they were satisfactory in the mid-term; C) The rate of reoperation and cardio-related death were analyzed by Kaplan-Meier survival estimate. They were not significantly different between the PP and TP groups. 
Table 1. Preoperative characteristics, echocardiographic parameters, and operative findings compared between PP and TP groups

\begin{tabular}{|c|c|c|c|}
\hline Age & $72.4 \pm 4.5$ & $64.1 \pm 14.0$ & .192 \\
\hline Body mass index & $1.5 \pm 0.2$ & $1.7 \pm 0.2$ & .049 \\
\hline Hypertension & $6 / 10(60 \%)$ & $11 / 22(50 \%)$ & .605 \\
\hline Chronic renal failure & $3 / 10(30 \%)$ & $3 / 22(14 \%)$ & .279 \\
\hline Chronic AF & $6 / 10(60 \%)$ & $6 / 22(27 \%)$ & .081 \\
\hline NYHA classification & $2.4 \pm 1.2$ & $1.7 \pm 1.0$ & .144 \\
\hline Reoperation & $1 / 10(10 \%)$ & $1 / 22(5 \%)$ & .561 \\
\hline Barlow's Disease & $0 / 10(0 \%)$ & $4 / 22(18 \%)$ & .156 \\
\hline LVDd (mm) & $56.1 \pm 9.8$ & $55.4 \pm 9.1$ & .791 \\
\hline LVDs (mm) & $41.0 \pm 10.6$ & $36.4 \pm 10.2$ & .116 \\
\hline IVS (mm) & $9.3 \pm 1.3$ & $10.0 \pm 1.3$ & .138 \\
\hline $\mathrm{LAD}(\mathrm{mm})$ & $50.0 \pm 15.3$ & $49.7 \pm 10.8$ & .760 \\
\hline SVI & $36.9 \pm 15.2$ & $30.1 \pm 19.0$ & .154 \\
\hline Mean PAP (mmHg) & $24.6 \pm 8.1$ & $18.1 \pm 7.2$ & .063 \\
\hline AR Grade & $1.3 \pm 1.2$ & $0.6 \pm 0.7$ & .229 \\
\hline MR Grade & $3.8 \pm 0.4$ & $3.9 \pm 0.3$ & .395 \\
\hline $\mathrm{RVol}(\mathrm{ml})$ & $63.7 \pm 17.3$ & $100.0 \pm 44.4$ & .012 \\
\hline Anterior Leaflet Angle & $18.9 \pm 20.9$ & $20.0 \pm 13.5$ & .629 \\
\hline Posterior Leaflet Angle & $49.4 \pm 21.7$ & $34.1 \pm 25.6$ & .132 \\
\hline \multicolumn{4}{|l|}{ Operative Findings } \\
\hline $\mathrm{A} 1$ & $0 / 10(0 \%)$ & $3 / 22(14 \%)$ & .227 \\
\hline A2 & $10 / 10(100 \%)$ & $11 / 22(50 \%)$ & .007 \\
\hline $\mathrm{A} 3$ & $2 / 10(20 \%)$ & $16 / 22(73 \%)$ & .006 \\
\hline P1 & $1 / 10(10 \%)$ & $2 / 22(9 \%)$ & .936 \\
\hline P2 & $0 / 10(0 \%)$ & $4 / 22(18 \%)$ & .156 \\
\hline P3 & $0 / 10(0 \%)$ & $4 / 22(18 \%)$ & .156 \\
\hline Operation Time (minutes) & $281.7 \pm 101.2$ & $277.9 \pm 119.6$ & .704 \\
\hline CPB Time (minutes) & $174.3 \pm 39.2$ & $195.0 \pm 62.3$ & .671 \\
\hline Clamp Time (minutes) & $109.4 \pm 27.0$ & $124.9 \pm 46.6$ & .540 \\
\hline Minimum Invasive Surgery & $8 / 10(80 \%)$ & $17 / 22(77 \%)$ & .865 \\
\hline Size of Artificial Ring & $29.6 \pm 3.0$ & $28.9 \pm 3.5$ & .941 \\
\hline Mitral Surgery Alone (cases) & 2 & 12 & \\
\hline
\end{tabular}


Table 1. Preoperative characteristics, echocardiographic parameters, and operative findings compared between PP and TP groups [cont.]

\begin{tabular}{l}
\hline \\
\hline + Aortic valve replacement \\
+ Aortic valve repair \\
+ Coronary artery bypass grafting \\
+ Tricuspid annuloplasty \\
+ Pulmonary vein isolation \\
+ Maze procedure \\
+ Aortic surgery \\
+ Atrial septal defect closure \\
which first was reported in 2011 and increasingly has been \\
recognized [Gertz 2011 ; Liang 2016 ]. Nevertheless, the fea- \\
tures and etiology of pseudoprolapse is not fully recognized, \\
and the ideal surgical procedure for pseudoprolapse cases has \\
not yet been determined. The present study conducted to \\
investigate the clinical findings of pseudoprolapse comparing \\
with true AML prolapse, classify the etiology of pseudopro- \\
lapse, and evaluate the effect of simple annular stabilization.
\end{tabular}

\section{MATERIALS AND METHODS}

Definition of pseudoprolapse and true prolapse of the AML: In the present study, pseudoprolapse is defined by the gap created by the leaflets without dropping into the left atrium (LA) beyond the anteroposterior annular line with eccentric MR flow directed to the posterior atrium (Figure 1A). True leaflet prolapse was diagnosed confirming the gap, which was created by the leaflets and the prolapsed leaflet dropping into the LA beyond the anteroposterior annular line.

Study population: One-hundred-twenty-two consecutive patients underwent surgery for severe $M R$ at our institute between January 2015 to July 2018. Of those, 74 patients with AML prolapse with or without posterior mitral leaflet (PML) prolapse were included.

Regarding the Carpentier's classification [Carpentier 1980], Type I (normal leaflet with annular dilatation) and Type III (restricted leaflet motion) cases were excluded in the present study. Additionally, patients who underwent concomitant operations of mitral valve replacement, active infectious endocarditis, and severe aortic regurgitation also were also excluded. Among the residual 32 cases, 10 were diagnosed as pseudoprolapse and classified as the Pseudoprolapse (PP) Group. The other 22 cases had obvious leaflet abnormalities and were classified as the True Prolapse (TP) Group. Pre- and postoperative parameters retrospectively were compared between the 2 groups.

Echocardiography: All patients underwent transthoracic echocardiography (TTE) preoperatively, postoperatively, and in the mid-term period. MR grade was assessed by measuring the color Doppler jet. MR grade is defined
Pseudoprolapse Group $(\mathrm{N}=10) \quad$ True Prolapse Group $(\mathrm{N}=22)$ $P$

$\begin{array}{ll}1 & 2 \\ 0 & 1 \\ 1 & 1 \\ 8 & 1 \\ 4 & 8 \\ 2 & 2 \\ 0 & 3 \\ 0 & 2 \\ & 4\end{array}$

as 0 ; non/trivial, 1 ; mild, 2 ; moderate, 3 ; moderate-severe, and 4 ; severe. The diameters of the mitral annulus were measured between A2 and P2 (anteroposterior diameter) annulus in both short and long axis view. Intercommissural diameter also was measured in short axis view. The leaflet angles of the AML and PML were measured between the annular line and coaptation point in the mid-systolic period.

The AFMR were defined by chronical atrial fibrillation (AF), the large LA, and 'hamstring' which is bending of the posterior wall inward toward the left ventricle (LV) cavity caused by mass effect exerted by the enlarged atrium [Yamano 2016; Silbiger 2019]. We also found the focal tethering of the posterior leaflet by the accessary papillary muscle, which we call "focal tethering."

Operative procedures: A minimally invasive approach through the fourth intercostal space was done in 25 cases. Median sternotomy also was done for the cases with complicated mitral lesion or concomitant with other procedures. Surgery was carried out under cardiac arrest with mild hypothermic cardiopulmonary bypass. The mitral valve was exposed through the incision in the right side of the LA. Simple annuloplasty procedure only was considered if it was diagnosed preoperatively as pseudoprolapse without evidence of abnormalities of the chordae tendineae or the papillary muscle. Full ring (Physio Annuloplasty Ring I; Edwards Lifesciences, CA, USA) annuloplasty was performed in all cases with the same size ring measured by the AML area. For the true AML prolapse, which had rupture or obvious elongation of chordae, the artificial chordae reconstruction was established followed by the annuloplasty. Saline injection testing was done several times during operation. Intraoperative transesophageal echocardiography (TEE) was performed to confirm the postoperative MR grade.

Statistical analysis: Continuous data are presented as the mean \pm SD. Mann Whitney test, Kruskal Wallis test, Friedman test, and Kaplan-Meier method were used for comparing variables. $P$ value of less than 0.05 was considered to be significant. All data were analyzed using SPSS (version 23; SAS Institute Inc, Cary, NC). 
Table 2. Analysis of cases of the PP Group $(\mathrm{N}=10)$

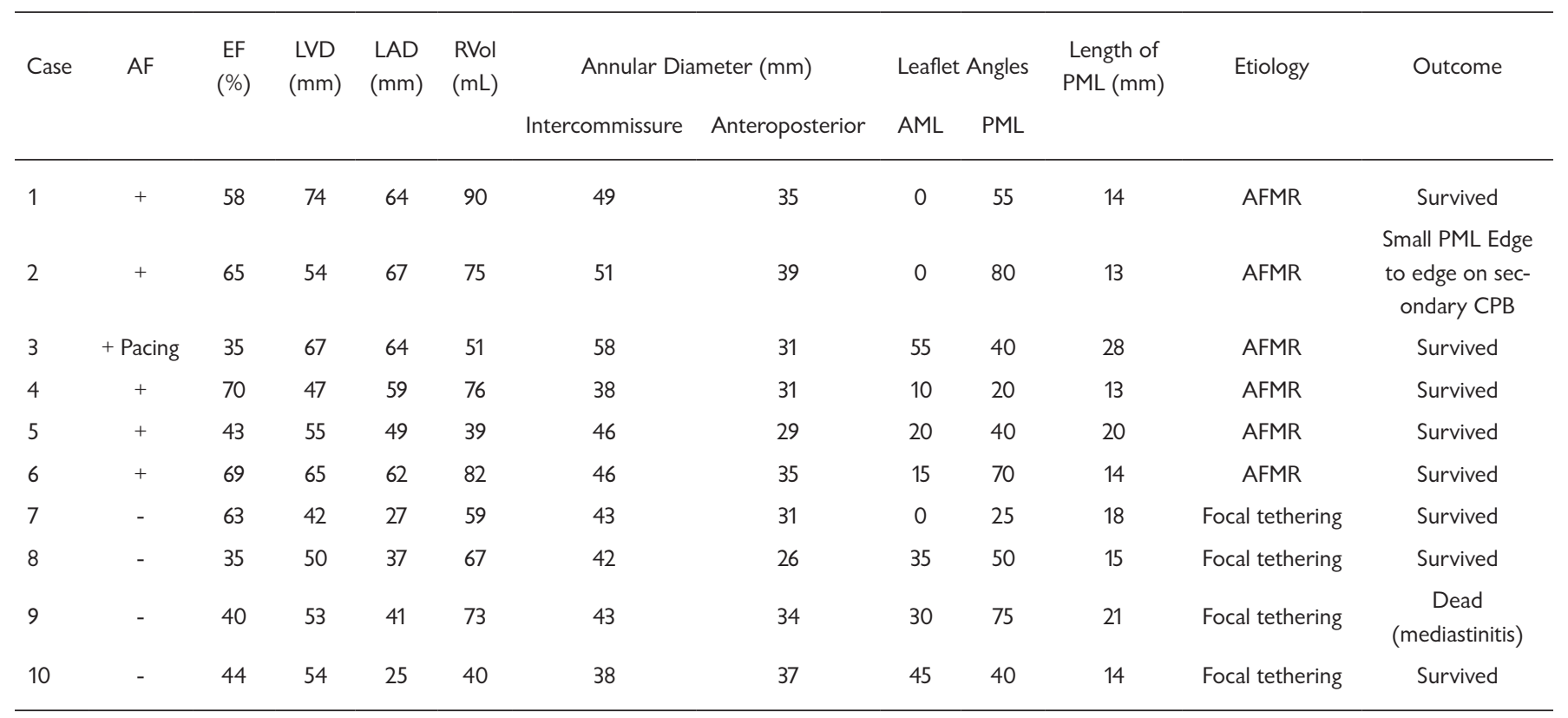

Case nos. 1-6 were considered to have AFMR. Case nos. 7-10 were considered to have focal tethering of the posterior LV wall.

MR: mitral regurgitation; AF: atrial fibrillation; AFMR: atrial functional mitral regurgitation; AML: anterior mitral leaflet; PML: posterior mitral leaflet; PP: pseudoprolapse; TP: true prolapse; TTE: transthoracic echocardiography; TEE: transesophageal echocardiography; EF: ejection fraction; LA: left atrium; LAD: left atrial dimension; LV: left ventricle; LVDd: left ventricular end-diastolic dimension; LVDs: left ventricular end-systolic dimension; IVS: interventricular septum; SVI: stroke volume index; PAP: pulmonary arterial pressure; RVol: regurgitant volume; RF: regurgitant fraction; ERO: effective regurgitant orifice; AR: aortic regurgitation; NYHA: New York Heart Association; CPB: cardiopulmonary bypass

\section{RESULTS}

Patient characteristics and echocardiographic measurements: Patient characteristics and preoperative echocardiographic data are shown in Table 1. Age, gender, rate of hypertension, chronical renal failure, chronic AF, and NYHA classification were not significantly different. Body mass index was greater in the TP Group. Patients in the PP Group had lower ejection fraction (EF) and lower regurgitant volume (RVol). Mitral annular diameter was not different between the 2 groups. The posterior leaflet angle of the PP Group tended to be bigger than those of the TP Group. Mean observation periods were $611 \pm 406$ and $574 \pm 380$ days in the PP and TP group, respectively.

Operative outcomes: Operative findings are shown in Table 1. All cases in the PP Group had eccentric jet originated from central regurgitation at A2 lesion as the main cause of MR. On the other hand, only half of the TP Group had A2 lesion. A3 lesion was much fewer in the PP Group than those in the TP Group. Around $80 \%$ of cases underwent minimally invasive cardiac surgery in both groups. The average size of artificial rings for the annuloplasty was not different significantly between the groups. Concomitant procedures were aortic replacement (3 cases), aortic repair (1 case), coronary artery bypass grafting (2 cases), tricuspid annuloplasty (16 cases), pulmonary vein isolation (6 cases), Maze procedure (5 cases), ascending aortic replacement (1 case), aortic root remodeling (1 case), and atrial septal defect closure (4 cases).
In both groups, MR grade were improved postoperatively, and maintained to be trivial by the mid-term (Figure 1B). One case in the PP Group needed edge-to-edge technique as an additional procedure on secondary cardiopulmonary bypass (CPB). The rates of the reoperation and cardio-related death were not significantly different between the 2 groups (Figure 1C).

Detailed characteristics of the Pseudoprolapse Group: All 10 cases in the PP Group are described in Table 2. Six cases were clarified as AFMR (Nos. 1-6) and had a large atrium (49-67 mm, $61 \mathrm{~mm}$ on average). All of cases of AFMR had atrial fibrillation and "hamstring." The annular diameter and angles of the AML and PML were not affected by AFMR. In Figure 2, panels $\mathrm{A}$ and $\mathrm{B}$ are the echocardiographic findings of AFMR case (No. 1 in Table 2), which show the large LA and MR going toward the posterior wall of the LA (Figure 2). Panel $C$ shows the surgical finding of case No. 1. The leaflets were not affected; the height of the AML and PML were not small, and coaptation was maintained under cardiac arrest. On the other hand, panels D and E show the echocardiographic findings of the other AFMR case (No. 2 in Table 2), which had a larger LA than case No. 1, and MR direction toward the posterior wall of the LA. Panel F shows the AML of case No. 2, which had smooth surface and enough height for coaptation. Panel G shows the PML of case No. 2, which was stiff and shortened. Consequently, case No. 2 needed secondary $\mathrm{CPB}$ conducted with additional edge-to-edge 


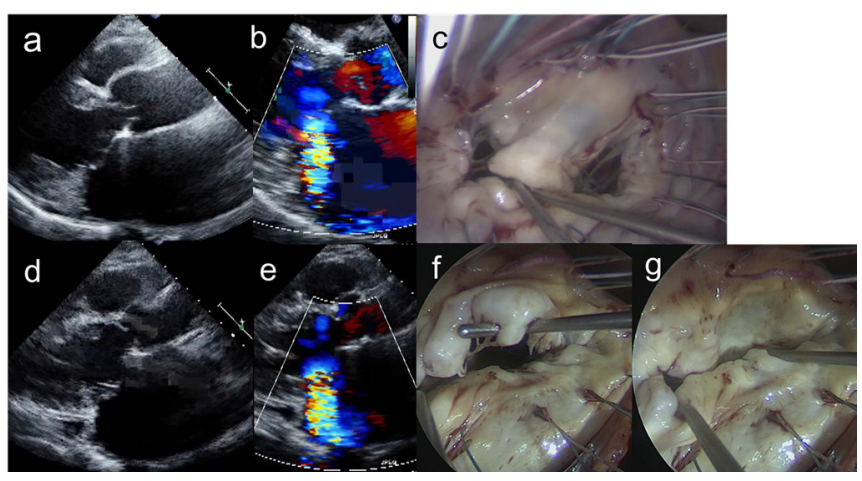

Figure 2. Echocardiographic and surgical findings. A) Echocardiographic findings of case No. 1 in Table 2 that shows the large LA; B) Echocardiographic findings of case No. 1 in Table 2; the regurgitation went toward the posterior wall; C) Surgical findings of case No. 1 in Table 2; the height of the AML and PML were satisfactory and almost the same; D) Echocardiographic findings of case No. 2 in Table 2, which shows the extremely large LA; E) Echocardiographic findings of case No. 2 in Table 2; the regurgitation went toward the posterior wall; F) Surgical findings of case No. 2 in Table 2; the surface of the AML was smooth, and the height was maintained; G) Surgical findings of case No.2 in Table 2. The PML was thicken and shortened.

technique after the primary simple annuloplasty because of insufficient coaptation due to the small, stiff, and shortened PML.

Four cases were considered to have focal PML tethering by accessary papillary muscle with short chordae directly from the posterior wall of the $L V$, which contributed severe MR (Nos. 7-10 in Table 2). EF was relatively low and the posterior leaflet angle was relatively high in the focal tethering cases than those in the AFMR cases.

\section{DISCUSSION}

The present study demonstrated that pseudoprolapse of the AML was characterized by having an eccentric jet directed to the posterior atrium, which originated from central regurgitation at $\mathrm{A} 2$ lesion as the main cause of $\mathrm{MR}$, low $\mathrm{EF}$, and low RVol, and could be treated with simple annuloplasty in most cases.

Pseudoprolapse cases with AFMR: Six cases in the PP Group were classified as AFMR defined by chronic AF, a large LA, and hamstring, which is bending of the posterior wall inward toward the LV cavity [Yamano 2016; Silbiger 2019]. MR due to mitral annular dilatation induced by LA enlargement is called AFMR, according to several reports [Gertz 2011; Liang 2016; Yamano 2016; Silbiger 2019; Takahashi 2015; Cong 2018; Ring 2014; Machino-Ohtsuka 2016; Saito 2018; Silbiger 2014; Pecha 2015]. The mechanism of AFMR are complex, including dilatation of the mitral annulus, flattening of the annular saddle shape, and greater leaflet tethering [Cong 2018]. Ring et al have demonstrated using $3 \mathrm{D}$ TEE that the mechanism of regurgitation in AFMR ultimately results in a loss of mitral leaflet apposition [Ring 2014]. The 4D analysis showed that the extremely dilated annulus in the patient with severe AFMR scarcely changed its area, during the cardiac cycle and annulus contraction [Machino-Ohtsuka 2016]. Typically, AFMR has a huge LA that causes the mounting of the posterior mitral annulus to the bended posterior inlet of the LV [Saito 2018], which is known as hamstring [Yamano 2016; Silbiger 2019]. Therefore, A2 of the AML is likely to be recognized as the main lesion of prolapse (pseudoprolapse) in AFMR cases. Also, it is considered that the posterior segment is less stiff than its anterior counterpart due to the fact that the posterior annulus is not a continuity of fibrous connective tissue, but fat tissue [Saito 2018]. In our cases, the hamstring was seen in all AFMR cases (Nos. 1-6 in Table 2). Hamstring caused the bent PML and prolapse of A2 lesion in the end-systolic period, which might be the reason why RVol was low in AFMR patients in the present study.

On the other hand, El Sabbagh et al reported that AFMR is regarded as secondary MR and classified into Carpentier's classification Type I, which is defined as normal leaflet and position [El Sabbagh 2018]. In AFMR, the mitral leaflet is normal in most cases, however, the basal position of the leaflet changes with the cardiac cycle because of the large LA and hamstring. The counterpart movement of the PML, which shows displacement of the basal leaflet position is similar to the leaflet movement of cases in Carpentier's Type II, which is defined as excess leaflet motion. AFMR cases may be difficult to be classified properly regarding Carpentier's classification. Further accumulation of publications about AFMR are needed for better understanding of this new defined disease.

Posterior leaflet angle in AFMR cases: AFMR is caused by mitral annular dilatation, but also by multiple factors, including the PML tethering. Associated with MR deterioration, the leaflet angle of PML became greater, however, the AML angle did not. Although the AML enlarged when the mitral annulus dilated, the PML failed to increase its area sufficiently [Machino-Ohtsuka 2016]. In the present study, the PML angle $(50.8 \pm 22.0)$ tended to be large compared with a normal leaflet angle of 34.4 \pm [Gelsomino 2008]. Annular diameter of AFMR were not significantly different from others.

Surgical procedure for AFMR: The prevalence with AFMR was reported to be $15.9 \%$ in hospitalized heart failure patients with AF [Saito 2018]. In the present study, 9.5\% (7 of 74 cases) of severe MR patients who underwent mitral repair were considered to have AFMR. AFMR patients were associated with a higher rate of a composite of cardiac death and readmission for heart failure compared with patients without MR [Saito 2018], therefore we recommend AFMR patients with severe MR to undergo mitral surgery. The leaflets of AFMR cases are usually smooth as normal, therefore, stabilization of the mitral annulus which excessively moves is the most important procedure. However, Takahashi et al reported a case of small size of the PML with AFMR [Takahashi 2015; Takahashi 2014]. In the present study, we experienced a case (No. 2 in Table 2) of small height of P2 lesion that was thicker than other lesions as shown in panel $\mathrm{G}$ in Figure 2. The case had an extremely huge LA and was thought to have long suffered from AFMR. Silbiger reported that AFMR has anatomical changes of the LA, due to longstanding alterations to the PML from the LA pressure and 
volume [Silbiger 2014], and which may change the PML to thicken and shorten. As the previous study, leaflet augmentation may serve as a useful adjunct to ring annuloplasty for the patients with marked leaflet tethering and a small PML [Silbiger 2019; Silbiger 2014]. In the present study, simple annuloplasty was not able to restore the MR with a small PML; it required leaflet repair including edge-to-edge technique or leaflet patch augmentation.

Kawazoe et al reported that the expanded LA compresses the posterobasal wall of the $\mathrm{LV}$ resulting in it bending inward, and the paradoxical movement of this segment causes lowoutput syndrome in MR cases with giant LA. Additionally, they suggested the plication technique for huge LA [Kawazoe 1983]. However, whether a huge LA needs to be resected is still controversial in AFMR cases with hamstring. Further study is needed.

The antiarrhythmic surgical procedure is necessary in almost all AFMR cases. In patients with successful AF ablation who remained in sinus rhythm at follow up, improvement in severity of MR was seen along with decreased LA size and mitral annular dimensions [Liang 2016]. However, in patients with a significantly enlarged LA diameter of more than $55 \mathrm{~mm}$, concomitant surgical ablation provided freedom from $\mathrm{AF}$ of $64.4 \%$ one year after the surgery. AF patients with a huge LA are likely to need additional catheter-based ablation to achieve satisfactory long-term results [Pecha 2015].

Pseudoprolapse cases caused by focal tethering: Four cases in the Pseudoprolapse Group were diagnosed as focal tethering of the posterior wall of the LV rather than AFMR because the motion of the posterior wall was slightly decreased compared with the other portion. The cases preoperatively were not considered Carpentier's classification Type III because the posterior wall, which caused tethering, was partially limited. Eccentric regurgitation jet caused by asymmetric coaptation is the reason of the AML pseudoprolapse as previously reported [Levi 2001; Agricola 2004; Zeng 2014]. These cases tended to have low EF compared with the cases of AFMR; this may affect the result as the PP Group had low EF in total. Otsuji et al reported that gradual deleterious $L V$ remodeling leads to dilatation of the mitral annulus as well as the posterior displacement of the subvalvular apparatus, resulting in excessive valvular tenting and malcoaptation [Otsuji 1997]. In the present study, the posterior leaflet angle of the focal tethering cases tended to be large, however, they had normal LV size, which is different from Carpentier's Type IIIb MR. Mitral leaflet is typically intact in the focal tethering cases, therefore, annular stabilization by ring is important. However, further remodeling following the LV dilatation has the possibilities of MR recurrence [Hashim 2012], so that additional techniques including leaflet patch augmentation can be considered. In addition, subvalvular structures should be observed taking variation of papillary muscle or chordae tendineae into account.

\section{LIMITATIONS}

The present study has several limitations. First, it was conducted as a single-center, retrospective, observational study with a small number of patients. However, this is the first report that focuses on evaluating and classifying pseudoprolapse cases, and verifies the ideal surgical procedure. Further study is needed with a large number of cases. Second, the TTE and TEE were not done by a single operator. Third, we could not show the details about the subvalvular structures, such as chordae tendineae and papillary muscles.

\section{CONCLUSION}

AML prolapse without dropping into the LA beyond the anteroposterior annular line could be defined as pseudoprolapse. This was characterized by an eccentric jet directed to the posterior atrium that originated from the central regurgitation at $\mathrm{A} 2$ lesion, low $\mathrm{EF}$, and low RVol, and it classified mainly into AFMR and focal tethering. Almost all cases could be treated by simple annuloplasty, but one case needed an additional leaflet technique.

\section{REFERENCES}

Agricola E, Oppizzi M, Maisano F et al. 2004. Echocardiographic classification of chronic ischemic mitral regurgitation caused by restricted motion according to tethering pattern. Eur J Echocardiogr 5:326-34.

Berdejo J, Shiota M, Mihara H, Itabashi Y, Utsunomiya H, Shiota T. 2017. Vena contracta analysis by color Doppler three-dimensional transesophageal echocardiography shows geometrical differences between prolapse and pseudoprolapse in eccentric mitral regurgitation. Echocardiography 34:683-689.

Carpentier A, Chauvaud S, Fabiani JN et al. 1980. Reconstructive surgery of mitral valve incompetence: ten-year appraisal. J Thorac Cardiovasc Surg 79:338-48.

Cong T, Gu J, Lee AP et al. 2018. Quantitative analysis of mitral valve morphology in atrial functional mitral regurgitation using real-time 3-dimensional echocardiography atrial functional mitral regurgitation. Cardiovasc Ultrasound 16:13.

El Sabbagh A, Reddy YNV, Nishimura RA. 2018. Mitral Valve Regurgitation in the Contemporary Era: Insights Into Diagnosis, Management, and Future Directions. JACC Cardiovasc Imaging 11:628-643.

Gelsomino S, Lorusso R, Caciolli S et al. 2008. Insights on left ventricular and valvular mechanisms of recurrent ischemic mitral regurgitationafter restrictive annuloplasty and coronary artery bypass grafting. J Thorac Cardiovasc Surg 136:507-18.

Gertz ZM, Raina A, Saghy L et al. 2011. Evidence of atrial functional mitral regurgitation due to atrial fibrillation: reversal with arrhythmiacontrol. J Am Coll Cardiol 58:1474-81.

Hashim SW, Youssef SJ, Ayyash B et al. 2012. Pseudoprolapse of the anterior leaflet in chronic ischemic mitral regurgitation: identification and repair. J Thorac Cardiovasc Surg 143:S33-7.

Kawazoe K, Beppu S, Takahara Y et al. 1983. Surgical treatment of giant left atrium combined with mitral valvular disease. J Thorac Cardiovasc $85: 885-892$.

Levi GS, Bolling SF, Bach DS. 2001. Eccentric mitral regurgitation jets among patients having sustained inferior wall myocardial infarction. Echocardiography 18:97-103. 
Liang JJ, Silvestry FE. 2016. Mechanistic insights into mitral regurgitation due to atrial fibrillation: "Atrial functional mitral regurgitation". Trends Cardiovasc Med 26:681-689.

Machino-Ohtsuka T, Seo Y, Ishizu T et al. 2016. Novel Mechanistic Insights Into Atrial Functional Mitral Regurgitation- 3-Dimensional Echocardiographic Study. Circ J 80:2240-8.

Otsuji Y, Handschumacher MD, Schwammenthal E et al. 1997. Insights from three-dimensional echocardiography into the mechanism of functional mitral regurgitation: direct in vivo demonstration of altered leaflet tethering geometry. Circulation 96:1999-2008.

Pecha S, Hakmi S, Subbotina I, Willems S, Reichenspurner H, Wagner FM. 2015. Concomitant surgical ablation for atrial fibrillation (AF) in patients with significant atrial dilation $>55 \mathrm{~mm}$. Worth the effort? J Cardiothorac Surg 10:165.

Ring L, Dutka DP, Wells FC, Fynn SP, Shapiro LM, Rana BS. 2014. Mechanisms of atrial mitral regurgitation: insights using 3D transoesophageal echo. Eur Heart J Cardiovasc Imaging 15:500-8.

Saito C, Minami Y, Arai K et al. 2018. Prevalence, clinical characteristics, and outcome of atrial functional mitral regurgitation in hospitalized heart failure patients with atrial fibrillation. J Cardiol 72:292-299.
Silbiger JJ. 2014. Does left atrial enlargement contribute to mitral leaflet tethering in patients with functional mitral regurgitation? Proposed role of atriogenic leaflet tethering. Echocardiography 31:1310-1.

Silbiger JJ. 2019. Mechanistic insights into atrial functional mitral regurgitation: Far more complicated than just left atrial remodeling. Echocardiography 36:164-169.

Takahashi Y, Abe Y, Sasaki Y et al. 2015. Mitral valve repair for atrial functional mitral regurgitation in patients with chronic atrial fibrillation. Interact Cardiovasc Thorac Surg 21:163-8.

Takahashi Y, Shibata T, Hattori K et al. 2014. Extended posterior leaflet extension for mitral regurgitation in giant left atrium. J Heart Valve Dis 23:88-90.

Yamano T, Furukawa K, Matoba S. 2016. "Hamstring Leaflet" Now Demonstrated to Be a Determinant of Functional Mitral Regurgitation in Patients With Atrial Fibrillation. Circ J 80:2098-9.

Zeng X, Nunes MC, Dent J et al. 2014. Asymmetric versus symmetric tethering patterns in ischemic mitral regurgitation: geometric differences from three-dimensional transesophageal echocardiography. J Am Soc Echocardiogr 27:367-75. 